\title{
Within-host competition drives energy allocation trade-offs in an insect parasitoid
}

\author{
J. Keaton Wilson ${ }^{\text {Corresp., } 1}{ }^{1}$ Laura Ruiz $^{2}{ }$ Goggy Davidowitz $^{1}$ \\ 1 Department of Entomology, University of Arizona, Tucson, Arizona, United States \\ 2 Department of Neuroscience, University of Arizona, Tucson, Arizona, United States \\ Corresponding Author: J. Keaton Wilson \\ Email address: keatonwilson@me.com
}

Organismal body size is an important biological trait that has broad impacts across scales of biological organization, from cells to ecosystems. Size is also deeply embedded in life history theory, as the size of an individual is one factor that governs the amount of available resources an individual is able to allocate to different structures and systems. A large body of work examining resource allocation across body sizes (allometry) has demonstrated patterns of allocation to different organismal systems and morphologies, and extrapolated rules governing biological structure and organization. However, the full scope of evolutionary and ecological ramifications of these patterns have yet to be realized. Here, we show that density-dependent larval competition in a natural population of insect parasitoids (Drino rhoeo: Tachinidae) results in a wide range of body sizes (largest flies are more than 6 times larger (by mass) than the smallest flies). We describe strong patterns of trade-offs between different body structures linked to dispersal and reproduction that point to life history strategies that differ between both males and females and individuals of different sizes. By better understanding the mechanisms that generate natural variation in body size and subsequent effects on the evolution of life history strategies, we gain better insight into the evolutionary and ecological impacts of insect parasitoids in tri-trophic systems. 
1

2

3

4

5

6

7

8

9

10

11

12

13

14

15

16

17

18

19

20

21

22

23

24

25

26

27

28

29

30

31

32

33

34

35

36

37

38

39

40

41

42

43

44

45
WITHIN-HOST COMPETITION DRIVES ENERGY ALLOCATION TRADE-OFFS IN AN INSECT PARASITOID

\author{
J. Keaton Wilson*,a Laura Ruiz ${ }^{\mathrm{b}} \&$ Goggy Davidowitz ${ }^{\mathrm{a}}$
}

a Department of Entomology, University of Arizona, 1140 E South Campus Drive, Forbes 410, PO Box 210036 Tucson, AZ 85721

${ }^{\mathrm{b}}$ Department of Neuroscience, University of Arizona, 1040 E 4th Street, PO Box 210077, Tucson AZ 85721

*Corresponding author - keatonwilson@me.com 
46

47

48

\section{Abstract}

Organismal body size is an important biological trait that has broad impacts across scales of 5 biological organization, from cells to ecosystems. Size is also deeply embedded in life history theory, as the size of an individual is one factor that governs the amount of available resources an individual is able to allocate to different structures and systems. A large body of work examining resource allocation across body sizes (allometry) has demonstrated patterns of allocation to different organismal systems and morphologies, and extrapolated rules governing biological structure and organization. However, the full scope of evolutionary and ecological ramifications of these patterns have yet to be realized. Here, we show that density-dependent larval competition in a natural population of insect parasitoids (Drino rhoeo: Tachinidae) results in a wide range of body sizes (largest flies are more than 6 times larger (by mass) than the smallest flies). We describe strong patterns of trade-offs between different body structures linked to dispersal and reproduction that point to life history strategies that differ between both males and females and individuals of different sizes. By better understanding the mechanisms that generate natural variation in body size and subsequent effects on the evolution of life history strategies, we gain better insight into the evolutionary and ecological impacts of insect parasitoids in tri-trophic systems.

67




\section{Introduction}

81

82 Body size is a biological trait that spans over 21 orders of magnitude (West, Brown, and Enquist 83 1999) with important impacts on chemical and physiological processes and inter- and intraspecific interactions in communities and ecosystems. Size can shape physiological processes like metabolism (West, Brown, and Enquist 1997) and is the outcome of complex genetic and developmental mechanisms (Davidowitz et al. 2003, 2012, 2016). Furthermore, biologists have long examined patterns of body size over large spatial scales (Blackburn, Gaston, and Loder 1999; Mousseau 1997), and examined how body size can affect complex community food webs (Jonsson, Cohen, and Carpenter 2005). Examining the relative size of different body parts (allometric scaling relationships (Gould 1966)) has become an important analytical tool alongside measurements of body size in diverse research topics including sexual selection (Emlen and Nijhout 2000; McCullough and Emlen 2013) physiological adaptation (Lane et al. 2018) and animal communication (Templeton, Greene, and Davis 2005). Together, body size and allometric scaling relationships not only provide a framework for conserved scaling rules that underlie developmental, physiological, and evolutionary processes (West, Brown, and Enquist 1997), but provide an opportunity to examine life history trade-offs, particularly in organisms like insects, where different body structures can act as proxies for investment into different life history strategies (reproduction, dispersal, information-acquisition and cognition).

At its core, life history theory seeks to determine the constraints on selection. Why doesn't selection drive all fitness related traits to high levels and how do organisms differ in rules that govern trade-offs (Stearns 1989; Fairbairn and Roff 2006)? Life history trade-offs (defined as life history traits that are negatively associated with each other; Zera and Harshman 2001) have been studied across a wide variety of taxa (Zera and Harshman 2001), including extensive work on insects, particularly Drosophila (Reznick 1985) and crickets (Mole and Zera 1994; Zera and Denno 1997), to determine constraints and mechanisms that govern optimal fitness strategies (Reznick 1985). Work in wing polymorphic crickets has demonstrated a strong trade-off between flight capability and fecundity (Roff 1986; Zera, Potts, and Kobus 1998), as well as the importance of nutrient acquisition in shaping trade-offs among traits (Zera and Brink 2000). Though there are many clear examples of trade-offs, results are often mixed as some traits 
111 demonstrate little or no trade-offs (McCullough and Emlen 2013; Fernández and Reynolds

112 2000; Tigreros and Davidowitz 2019). While measurements of allometry involve size (mass) of

113 particular structures, these measurements can prove problematic when addressing life history

114 trade-offs, because allocation costs can be masked in low-mass but energy-dense tissues (Zera

115 and Harshman 2001). Energy provides a common currency with which to compare allocation to

116 different tissues because of energetic differences among molecular building-blocks (i.e. an

117 abdomen and thorax of similar weight may not accurately reflect a similar energetic allocation of

118 resources, as the abdomen may contain a high proportion of energetically dense lipids). Here,

119 we use a combination of weight and energy measurements to examine allocation to different

120 body structures in a parasitoid fly (Drino rhoeo).

121

122 Tachinid flies (Diptera: Tachinidae) are internal parasitoids of arthropods and typically attack

123 larval stages of herbivorous insects (Stireman, O'Hara, and Wood 2006). Tachinids are an

124 understudied yet ecologically important group with approximately 10,000 described species that

125 likely have powerful roles in shaping communities of plants and insects (Stireman, O'Hara, and

126 Wood 2006; Wilson and Woods 2015). Drino rhoeo is a good model for examining trade-offs in

127 resource allocation and patterns in body size because it is a gregarious parasitoid (multiple

128 larvae develop together within a single host) with large variation in cohort size (here meaning

129 groups of $D$. rhoeo larvae developing inside a single host, typically 8-50 larvae within a given

130 host (Wilson and Woods 2015)). This variation means that there is likely competition for host

131 resources at high densities, which can lead to naturally-occurring variation in nutrient acquisition

132 available to larvae, resulting in population-level variation in body size and trait size. (Zera and

133 Harshman 2001).

134

135

Drino rhoeo attacks caterpillars of the hawkmoth Manduca sexta and Manduca

136

quinquemaculata (Bernays and Woods 2000; Mira and Bernays 2002; Wilson and Woods 2015)

137 frequently at our field site in southeastern Arizona (near Portal and the Chiricahua mountains).

138 Female flies target fourth- and fifth-instar caterpillars, laying eggs on the surface of the

139 caterpillar after which fly larvae shortly (within 20 minutes) emerge and burrow into the

140 caterpillar hemocoel where they grow and develop. Developing tachinid larvae typically

141 completely consume the bodies of their hosts, resulting in host-death. Previous work has

142 demonstrated that $D$. rhoeo can have a strong impact on the growth and development of hosts

143 post-parasitization, affecting growth, weight and feeding habits (Wilson and Woods 2015) which

144 may, in turn, affect the amount of resources available to the parasitoids. 
146 Here, we use data from a natural population of $D$. rhoeo to address three main questions: (1)

147 does larval competition and host quality drive variation in adult parasitoid body size, (2) what are

148 the energy allocation strategies to different parasitoid body structures (heads, abdomens,

149 thoraces, wings and legs) and how do they vary with parasitoid body size and sex and (3) are

150 there allocation trade-offs among body structures that act as proxies for different life history

151 strategies? To our knowledge, this if the first study that examines larval resource competition in

152 tachinids with an emphasis on energy allocation among different body structures relative to size

153 (resource competition has been shown by others: Allen, Geoff and Hunt 2001; Welch 2006,

154 Lehmann 2008) and to extend these effects to patterns of life history trade-offs.

155 Methods

156 Host and parasitoid collection

157 Thirty-two M. sexta larvae in the 4th or 5th (final) instar were collected from the field near Portal, 158 Arizona ( $40 \mathrm{~km}$ radius) in August of 2017. Past work with Drino rhoeo showed that 159 parasitization rate was high $(\sim 44 \%)$ in the field for $M$. sexta in 4 th and 5 th-instar stages (Wilson 160 and Woods 2015), so we collected a mixture of individuals that appeared healthy, and some 161 that appeared to have been attacked by parasitoids (e.g. dried gut fluids on the skin, melanized 162 spots where fly larvae burrowed inside the caterpillar [Woods and Wilson, unpublished]).

163 Larvae were raised together in a large plastic bin and fed cuttings from local Datura wrightii, 164 their main host plant. Once larvae began to wander (Dominick and Truman 1984), they were 165 placed in individual plastic cups $(13 \mathrm{~cm} \times 12 \mathrm{~cm} \times 14 \mathrm{~cm})$ filled with soil. Larvae were allowed to 166 burrow and begin pupation and were transported back to the University of Arizona in Tucson 167 where they were kept in an experimental greenhouse for the duration of their development.

168

169

170

171

172

173

174

\section{Head-capsule width as a measure of host quality}

Here, we define host larval quality as the energy potential of a host to developing parasitoid larvae. Larval mass is not a good measure of larval quality, because growth, feeding and development are all affected by parasitism (Wilson and Woods 2015), so we measured headcapsule width of hosts (all $5^{\text {th }}$-instar) as a proxy to estimate larval quality to developing flies. We examine the relationship between mass and head-capsule width in more detail in the Results section. 
175 Fly emergence, weights and calorimetry

176 Seven of the thirty-two caterpillars (22\%) collected were parasitized by Drino rhoeo and had

177 successful fly emergence. Flies were allowed to emerge in the small plastic cups containing

178 individual caterpillars described above. Once initial fly emergence was observed, we waited 48

179 hours to allow all individuals to successfully emerge from the soil, and then placed the cups in a

$180-20^{\circ} \mathrm{C}$ freezer. Once flies were frozen, we transferred them to individually-labeled vials and

181 scored sex using a dissecting scope to determine the presence of sexual patches on the ventral

182 portion of the last abdominal segment on males. Files were sectioned into different body

183 structures (head, abdomen, thorax, wings and legs) using a combination of scalpel, probe and

184 forceps under a dissecting scope. Sectioned body structures were moved to small metal

185 containers and placed in a drying oven at $\sim 45^{\circ} \mathrm{C}$ for 48 hours before being placed back into

186 vials and frozen again at $-20^{\circ} \mathrm{C}$ until further processing. Dried individual body sections were

187 weighed on a microbalance (Mettler Toledo XS3DU), with legs and wings being weighed

188 together. In total, we collected 104 individual flies from the 7 hosts, though not all flies were

189 used in all subsequent analyses because of some loss of body structures during the weighing or

190 bombing process.

191

192 We used a Parr 6200 bomb calorimeter to determine energy content of heads, thoraces and

193 abdomens. Because our calorimeter is designed to be used on larger tissue, we generated

194 calibration curves for predicting caloric content based on tissue type and weight to be able to

195 extrapolate to small weights (and respectively small energy content) that are below the

196 threshold of detection for our calorimeter. Individual structures were mixed into different groups

197 ranging from 1 to 10 structures from individual flies per bin (depending on the size of the tissue:

198 more heads were needed to get to measurable weights than abdomens, because heads are

199 smaller than abdomens) and separated by sex. We also varied the number of structures in

200 binned groups to achieve enough variation in weight to generate accurate calibration curves.

201 Binned tissue samples were weighed and placed in a crucible. We added $0.7 \mathrm{~mL}$ of mineral oil

202 to samples as a heat spike (to increase the total energy content to a level readable by the

203 machine). This method of measuring small samples is a standard procedure and the Parr

204 calorimeter software automatically accounts for the mineral oil spike.

205

206 Zera and Harshman (2001) emphasize that to establish a physiological tradeoff among body

207 functions, there is a need to establish that a specific resource (such as a specific lipid, protein or

208 carbohydrate) is used by both functions which requires tracking the common resource in both

Peer] reviewing PDF | (2019:08:40077:3:0:NEW 17 Feb 2020) 
209 functions. In this study we are interested in total amount of resources allocated among functions

210 and not a specific resource. The use of energy as the common currency encompasses all

211 resources allocated to a function and allows for identifying physiological allocation tradeoffs

212 among functions. We note that this method cannot measure the cost of building a structure, just

213 the energy content of the resources that are in the structure.

215 We created tissue-specific calibration curves to generate estimates of the calorimetric content of 216 different tissues based on weight. We focused on the three main body segments (heads,

217 thoraces and abdomens). To generate curves, we fit linear regression models for each tissue 218 type that were forced through the origin. All models had good predictive power $(p<0.05$ for all 219 models), though the predictive power for estimating the energetic content of heads $\left(R^{2}=0.58\right)$ 220 was less than for thoraces $\left(R^{2}=0.90\right)$ and abdomens $\left(R^{2}=0.91\right)$ because of smaller sample 221 sizes (it takes many more fly-heads to generate the measurable weights than it does thoraces 222 or abdomens) and more variation in measurements at smaller weights. We did not have enough 223 flies to generate separate calibration curves for males and females, so all bins consisted of flies 224 from a single sex, and calibration curves are tissue-specific but not sex specific, which might 225 226 result in additional variation in extrapolations if there were large differences between the weightenergy correlations of different sexes.

\section{Head capsule and host tissue calorimetry}

228 To determine the relationship between head-capsule width and the usable energetic content of 229 hosts, we used late 5th-instar M. sexta caterpillars from our colony in Tucson, AZ. We 230 measured head-capsule width and then froze caterpillars in a $-20^{\circ} \mathrm{C}$ freezer. We then thawed 231 and separated caterpillar structures into two groups: skin and head-capsule and internal tissues 232 that would be available to developing parasitoids (this included hemolymph, tracheae, muscle, 233 but not gut tissue or any remaining food). We dried samples for each caterpillar in a drying oven 234 at $\sim 45^{\circ} \mathrm{C}$ for a minimum of 48 hours. We crushed dried tissue samples and split samples that 235 were too large for our calorimeter into three sub-samples before bombing. We used a bomb 236 calorimeter (Parr 6200 - methods described above) with a $600 \mu \mathrm{L}$ mineral oil spike to measure 237 the energetic content of each sub-sample before combining for further analysis. 


\section{Data Analyses}

239 All analyses were performed in R (Version 3.5.0 "Joy in Playing", www.r-project.org). We used

240

241

242

243

244

245

246

247

248

249

250

251

252

253

254

255

256

257

258

259

260

261

262

263

264

265

266

267

268 linear mixed effects models ( $n / m e$ ) for modeling the relationship between adult fly size, host quality, cohort size and sex and AIC scores for model comparison and selection. Additional packages (effects, piecewiseSEM) were used to generate population level trend lines and marginal and conditional $R^{2}$ values. In spite of our limited sample size of hosts $(n=7)$, we included host as a random effect (with random intercepts) to help control for variance in conditions among hosts. Additionally, we used ordinary least squares (OLS) regression for examining allometric relationships between body weight and tissue weight and energetic content and AIC scores for model comparison and selection (Supplementary Table 1). Though some researchers have advocated the use of RMA regression (reduced-major axis regression), recent work has shown that OLS regression is better suited in many cases, especially those similar to ours where there is comparatively little measurement error (Kilmer and Rodríguez 2017). In comparisons of relative mass and energy allocation, polynomial models were fit where appropriate. All data and code are archived and available on Zenodo (doi:10.5281/zenodo.3356991).

\section{Results}

\section{Cohort size, host quality and adult fly weight}

On average, adult fly body size did not differ between males and females (males $=8.74 \pm 3.58$ $\mathrm{mg}$, females $\left.=8.51 \pm 3.73 \mathrm{mg} ; F_{1,90}=0.091, p=0.7641\right)$. On average, adult fly dry weight decreased with increasing cohort size (Figure 1a), with an average fly from the largest cohort (38 flies) weighing $36 \%$ of an average fly from the smallest cohort (2 flies). Additionally, adult fly dry weight increased with increasing host quality (head-capsule width; Figure 1b). Overall, the best model that explained adult fly weight was one that included cohort size and host quality additively (Table 1-2) with host included as a random effect (random intercepts). We also performed a multiple linear regression on a reduced number of data points $(n=7)$ that were average fly-weight values for each cohort, to confirm the biological pattern we show here and reaffirm that the linear-mixed effects model framework is accounting for any pseudo-replication of sampling multiple flies within a single host. This analysis showed that similar significant effects of cohort size $(p=0.05)$ and host head capsule width $(p=0.03)$ with good predictive power $\left(p=0.006, R^{2}=0.76\right)$. Head-capsule width is a frequently-used proxy for body size, and 
269 typically has strong positive correlations with body size (Smock 1980; Potter, Davidowitz, and

270 Woods 2011; D'Amico, Davidowitz, and Nijhout 2001) - it is also the best measurement of host-

271 quality in this system because of the complex interactions between parasitism, host-feeding and

272 body size (Wilson and Woods 2015). We found some support that head-capsule width is

273 positively associated with wandering-weight $\left(F_{1,5}=6.961, p=0.046, R^{2}=0.498\right)$ in $M$. sexta

274 caterpillars in the field, despite a relatively small sample size and that caterpillars were

275 parasitized by varying numbers of tachinid larvae. Additionally, in a series of measurements on

276 lab-reared $M$. sexta, we found that pre-wander weight was positively correlated with the caloric

277 content of hosts excluding skin and the gut $\left(F_{1,13}=54.01, p<0.0001, R^{2}=0.79\right)$. Together,

278 these data suggest that head-capsule width functions as a good proxy for both body size and

279 caloric content available to developing tachinids.

280 Size-relative allocation to different body structures

281 Overall, flies had relatively smaller heads as size increased, with no difference between males

282 and females $(t=1.102, p=0.273)$, though the best fit model for these data was a $2 n d-o r d e r$

283 polynomial, with a slight increase in investment in heads at large body sizes $\left(F_{2,89}=51.8, p<\right.$ $0.001, R^{2}=0.53$; Figure 2a, Supplementary Table 1). Normalized wing weight (scaled to individual body size - we use this definition of normalized throughout) decreased with body size $286\left(F_{2,89}=10.74, p=0.001, R^{2}=0.09\right)$, with no differences between the sexes $(t=-0.768, p=$ 2870.444 ; Figure $2 b)$. Normalized leg weight decreased linearly with body size $\left(F_{2,89}=5.169, p=\right.$ $\left.2880.008, R^{2}=0.50\right)$, and while there was a significant effect of $\operatorname{sex}(t=-2.617, p=0.01)$, the effect 289 size was small, with little difference between males and females, except only at small body 290 sizes (Figure 2c). Normalized thorax weight was best fit with a polynomial model that included 291 sex as an additive effect $\left(F_{3,88}=35.98, p<0.001, R^{2}=0.54\right)$ - relative thorax weights were 292 highest in medium-sized flies, and were higher overall in males $(t=7.909, p<0.001)$, with the greatest difference between males and females occurring in medium-sized flies (Figure 2d). Finally, normalized abdomen weight increased linearly with fly weight in both males and females $\left(F_{2,89}=65.18, p<0.001, R^{2}=0.56\right)$, though females showed increased relative investment across all body weights $(t=-8.399, p<0.001$; Figure $2 e)$.

\section{Energy tradeoffs and comparisons among body segments}

298 Body segments differed in their average energy content with abdomens being the most energy dense at $4.36 \pm 0.44$ calories $/ \mathrm{mg}$, followed by thoraces at $4.05 \pm 0.33$ calories $/ \mathrm{mg}$, followed by 
300 heads at $1.96 \pm 0.17$ calories $/ \mathrm{mg}$. We compared the percentage of calories devoted to each

301 body segment for male and female flies across body sizes and found strong potential trade-offs

302 between thoraces and abdomens in both males and females, though the pattern is strongest for

303 male flies of moderate size (Figure 3a). We note here that while a negative correlation between

304 two traits has often been used as evidence for trade-offs (e.g. Zera and Harshman 2001;

305 Stearns 1989), the interactions among traits are complex, and indirect effects may impact

306 negative correlations. We also compared the relative amount of energy devoted to one segment

307 after controlling for the total energy content of the three main body segments. Both male and

308 female flies showed a strong negative correlation between the relative amount of energy

309 invested in thoraces and abdomens $\left(F_{1,99}=1983, p<0.001, R^{2}=0.952\right.$; Figure $\left.3 b\right)$, with no

310 difference between males and females $(p=0.209)$. There was no significant pattern of trade-

311 offs between heads and thoraces or heads and abdomens for male or female flies $\left(F_{1,99}=\right.$

$\left.3121.225, p=0.271 ; F_{1,99}=1.187, p=0.279\right)$.

\section{Discussion}

314 Drino rhoeo show clear indications of strong density-dependent larval competition (Figure 2),

315 similar to other gregarious insect parasitoids (Taylor 1988; Harvey 2000; Allen and Hunt 2001).

316 This competition (in combination with variation in host quality; Figure $2 \mathrm{~b}$, Table 1), leads to a

317 wide range of body sizes that is naturally occurring, and is not sexually dimorphic (Figure 1). In

318 spite of a low sample size of hosts $(n=7)$, we found strong relationships between cohort size and

319 adult fly weight, as well as moderate impacts of host quality on adult fly weight. The evolutionary

320 and ecological implications of density-dependent larval competition in insect parasitoids have

321 been examined only in hymenopteran parasitoids previously (Nicol and Mackauer 1999; Milonas

322 2005; Sykes et al. 2007). Our results presented here pave the way for further work examining

323 tradeoffs between immune function and larval competition, female tachinid oviposition

324 strategies, and optimal brood size in non-hymenopteran parasitoids.

325

326 Allometric scaling relationships of the mass of tissues provide insight into how investment in

327 structures change across body sizes and sexes. Flies invested in relatively larger wings, legs,

328 and heads at smaller body sizes while investing in relatively larger abdomens at high body sizes

329 and large thoraces at moderate body sizes (Figure 2). Male and female flies differed markedly in

330 their allocation to thoraces and abdomens with males investing relatively more in thoraces and

331 females investing relatively more in abdomens (Figure $2 d-e$ ). Fly legs, wings and heads showed 
332 hypoallometric scaling relationships (scaling coefficients of $0.709,0.559,0.68$, respectively;

333 Supplemental Figure 1), while thoraces for both males and female flies were isometric (scaling

334 coefficient of 0.986). Abdomens for both males and females were hyperallometric (scaling

335 coefficient of 1.236), mirroring previous work in other insects (Wickman and Karlsson 1989).

336

337 Both male and female flies showed strong trade-offs between abdomens and thoraces in both

338 mass (Figure 2) and energetic content (Figure 3b). Though this trade-off is consistent across

339 body sizes, relative allocation to each structure is markedly different between males and

340 females and across body sizes (Figure 3a). As a group, the relative investment into abdomens

341 or thoraces falls along a clear gradient - with females devoting energy to abdomens at the

342 expense of thoraces, while the opposite is true for males (Figure 3b). Though the majority of

343 flies follow this allocation rule, a few small male flies in our study demonstrate a different

344 allocation strategy - less investment overall to abdomens and thoraces while devoting more

345 energy to the head (Figure 3b). Investment in thoraces (a proxy for dispersal, though many

346 insects are capable of resorbing wing musculature as adults (Stjernholm, Karlsson, and Boggs

347 2005; Boggs 2009)) is favored at small and moderate body sizes, while equal investment in

348 thoraces and abdomens (reproduction and storage) is favored at large body sizes. These

349 patterns are magnified in male flies, where some individuals invest up to $80 \%$ of the total

350 energetic content of the three main body segments into their thoraces, and dampened in

351 females. There is evidence in other insect systems that increased allocation to thoraces is

352 correlated with higher flight performance (Berwaerts, Van Dyck, and Aerts 2002; Karlsson and

353 Johansson 2008), though the energy allocation to thoraces we present here may also represent

354 a minimum threshold required to produce functional flight. Researchers have also shown that

355 increased allocation to abdomens is correlated with increased fecundity (Wickman and Karlsson

356 1989; Griffith 1994; Preziosi et al. 1996). Here, flies demonstrate strategies that closely match

357 predictions made by life history theory for income-breeding insects (Davis et al. 2016) where

358 dispersal and mobility are favored in poor quality environments (tachinids are likely income

359 breeders that feed on nectar and pollen (Gilbert 1998; Tooker, Hauser, and Hanks 2006) and

360 can ameliorate larval nutritional deficits as adults by being able to find high-quality resource

361 sites) while a bigger relative investment in reproduction or energetic storage can be allocated in

362 high quality larval environments (Boggs 2009). These allocation patterns are likely magnified in

363 males and dampened in females because of heightened energy requirements for reproductive

364 investment and oviposition success (Reznick 1985). 


\section{Conclusions}

366 In conclusion, we show that larval competition and variation in host quality generate a range of

367 body sizes in a population of insect parasitoids which in turn leads to different patterns of

368 allocation to thoraces and abdomens across body sizes and between sexes. These strategies

369 point to strong trade-offs between body segments tied to reproduction and dispersal. By better

370 understanding the mechanisms that drive allocation to different tissues across body sizes and

371 between sexes, particularly in under-studied species with potentially large ecological effects, we

372 gain deeper insight into the evolution and ecology of tri-trophic systems and the underlying

373 drivers of life history strategies.

\section{Acknowledgements}

375 We'd like to thank the director of the Southwestern Research Station and station employees for their help, as well as Cristina Francois, Lennie Park, Natasha Tigreros and Meck Slagle for their input on these data, analyses and presentation. We'd also like to thank Heather Costa for her support in the lab and for input on data, analyses and presentation.

\section{9}

\section{References}

380

381

382

383

384

385

386

387

388

389

390

391

392

393

394

Allen GR, Hunt J. 2001. Larval Competition, Adult Fitness, and Reproductive Strategies in the Acoustically Orienting Ormiine Homotrixa Alleni (Diptera: Tachinidae). Journal of Insect Behavior 14 (3): 283-97.

Bernays EA, Woods, HA. 2000. Foraging in nature by larvae of Manduca Sexta - Influenced by an endogenous Oscillation." Journal of Insect Physiology 46 (5): 825-36.

Berwaerts, K, H Van Dyck, P Aerts. 2002. Does Flight Morphology Relate to Flight Performance? An Experimental Test with the Butterfly Pararge Aegeria. Functional Ecology $16(4): 484-91$.

Blackburn, TM, K,. Gaston, and N Loder. 1999. Geographic Gradients in body size: a clarification of Bergmann's Rule Diversity \& Distributions 5 (4): 165-74.

Boggs, CL. 2009. Uderstanding insect life histories and senescence through a resource allocation lens. Functional Ecology 23 (1): 27-37.

D’Amico, LJ, Davidowitz, G, Nijhout, HF. 2001. The Developmental and Physiological Basis of Body Size Evolution in an Insect. Proceedings. Biological Sciences 268 (1476): 1589-93.

Davidowitz, G, D’Amico, LJ, Nijhout, HF. 2003. Critical Weight in the Development of Insect 
395

396

397

398

399

400

401

402

403

404

405

406

407

408

409

410

411

412

413

414

415

416

417

418

419

420

421

422

423

424

425

426

427

428

Body Size. Evolution and Development 5 (2): 188-97.

Davidowitz G, Nijhout HF, Roff, DA. 2012. Predicting the response to simultaneous selection: genetic architecture and physiological constraints. Evolution 66:2916-2928.

Davidowitz, G, Roff, DA, Nijhout, HF. 2016. Synergism and antagonism of proximate mechanisms enable and constrain the response to simultaneous selection on body size and development time: an empirical test using experimental evolution. The American Naturalist 188: 499-520.

Davis RB, Javoiš, ., Kaasik, A, Õunap, E, Tammaru, T. 2016. An ordination of life-histories using morphological proxies: capital vs income breeding in insects. - Ecology , 97: 21122124

Dominick OS, Truman JW. 1984. The Physiology of Wandering Behaviour in Manduca Sexta. I. Temporal Organization and the Influence of the Internal and External Environments. The Journal of Experimental Biology 110: 35-51.

Emlen DJ, Nijhout, HF. 2000. The development and evolution of exaggerated morphologies in insects. Annual Review of Entomology 45: 661-708.

Fairbairn DJ, Roff, DA. 2006. The quantitative genetics of sexual dimorphism: assessing the importance of sex-linkage. Heredity 97 (5): 319-28.

Fernández RJ, Reynolds, JF. 2000. Potential Growth and Drought Tolerance of Eight Desert Grasses: Lack of a Trade-Off? Oecologia 123 (1): 90-98.

Gilbert F. 1998. Functional, Evolutionary and Ecological Aspects of Feeding-Related Mouthpart Specializations in Parasitoid Flies. Biological Journal of the Linnean Society. Linnean Society of London 63 (4): 495-535.

Gould SJ. 1966. Allometry and Size in Ontogeny and Phylogeny. Biological Reviews of the Cambridge Philosophical Society 41 (4): 587-640.

Griffith H. 1994. Body Elongation and Decreased Reproductive Output within a Restricted Clade of Lizards (Reptilia: Scincidae). Journal of Zoology 233 (4): 541-50.

Harvey JA. 2000. Dynamic Effects of Parasitism by an Endoparasitoid Wasp on the Development of Two Host Species: Implications for Host Quality and Parasitoid Fitness. Ecological Entomology 25: 267-78.

Jonsson T, Cohen JE, Carpenter SR. 2005. Food Webs, Body Size, and Species Abundance in Ecological Community Description. In Advances in Ecological Research, 1-84.

Karlsson B., Johansson A. 2008. Seasonal Polyphenism and Developmental Trade-Offs between Flight Ability and Egg Laying in a Pierid Butterfly. Proceedings of the Royal Society B: Biological Sciences 275 (1647): 2131-36. 
429

430

431

432

433

434

435

436

437

438

439

440

441

442

443

444

445

446

447

448

449

450

451

452

453

454

455

456

457

458

459

460

461

462

Kilmer JT, Rodríguez, RL. 2017. Ordinary Least Squares Regression Is Indicated for Studies of Allometry. Journal of Evolutionary Biology 30 (1): 4-12.

Lane SJ, Moran AL, Shishido CM, Tobalske BW, Woods HA. 2018. Cuticular Gas Exchange by Antarctic Sea Spiders. The Journal of Experimental Biology 221 (Pt 8). https://doi.org/10.1242/jeb.177568.

Lehmann, GU. (2008). How different host species influence parasitism patterns and larval competition of acoustically-orienting parasitoid flies (Tachinidae: Ormiini). Animal Behavior: New Research, 93-132.

McCullough EL, Emlen DJ. 2013. Evaluating the Costs of a Sexually Selected Weapon: Big Horns at a Small Price. Animal Behaviour 86 (5): 977-85.

Milonas PG. 2005. Influence of Initial Egg Density and Host Size on the Development of the Gregarious Parasitoid Bracon hebetor on Three Different Host Species. Biocontrol 50 (3): 415-28.

Mira A, Bernays EA. 2002. Trade-Offs in Host Use by Manduca Sexta: Plant Characters vs Natural Enemies. Oikos 97 (3): 387-97.

Mole S, Zera AJ. 1994. Differential Resource Consumption Obviates a Potential FlightFecundity Trade-Off in the Sand Cricket (Gryllus firmus). Functional Ecology 8 (5): 573.

Mousseau TA. 1997.“Ectotherms Follow the Converse to Bergmann's Rule. Evolution; International Journal of Organic Evolution 51 (2): 630.

Nicol CMY, Mackauer M. 1999. The Scaling of Body Size and Mass in a Host-Parasitoid Association: Influence of Host Species and Stage. Entomologia Experimentalis et Applicata 90 (1): 83-92.

Potter KA, Davidowitz G, Woods HA. 2011. Cross-Stage Consequences of Egg Temperature in the Insect Manduca Sexta. Functional Ecology. https://doi.org/10.1111/j.13652435.2010.01807.x.

Preziosi, RF, Fairbairn DJ, Roff DA, Brennan JM. 1996."Body Size and Fecundity in the Waterstrider Aquarius remigis: A Test of Darwin's Fecundity Advantage Hypothesis. Oecologia 108 (3): 424-31.

Reznick, D. 1985. Costs of Reproduction: An Evaluation of the Empirical Evidence. Oikos 44 (2): 257.

Roff, DA. 1986. The Evolution of Wing Dimorphism in Insects. Evolution; International Journal of Organic Evolution 40 (5): 1009.

Smock, LA. 1980. Relationships between Body Size and Biomass of Aquatic Insects." Freshwater Biology. https://doi.org/10.1111/j.1365-2427.1980.tb01211.x. 
463 Stearns SC. 1989. Trade-Offs in Life-History Evolution. Functional Ecology 3 (3): 259.

464

465

466

467

468

469

470

471

472

473

474

475

476

477

478

479

480

481

482

483

484

485

486

487

488

489

490

491

492

493

494

495

496

Stireman JO, O'Hara JE, Wood zfm. 2006. Tachinidae: Evolution, Behavior, and Ecology.” Annual Review of Entomology 51 (January): 525-55.

Stjernholm F, Karlsson B, Boggs CL. 2005. Age-Related Changes in Thoracic Mass: Possible Reallocation of Resources to Reproduction in Butterflies. Biological Journal of the Linnean Society. Linnean Society of London 86 (3): 363-80.

Sykes EM, Innocent TM, Pen I, Shuker DM, West SA. 2007. Asymmetric Larval Competition in the Parasitoid Wasp Nasonia vitripennis: A Role in Sex Allocation? Behavioral Ecology and Sociobiology 61 (11): 1751-58.

TammaruT, Haukioja E. 1996. Capital Breeders and Income Breeders among Lepidoptera: Consequences to Population Dynamics. Oikos 77 (3): 561.

Taylor AD. 1988. Host Effects on Larval Competition in the Gregarious Parasitoid Bracon Hebetor. The Journal of Animal Ecology 57 (1): 163.

Templeton CN Greene E, Davis K. 2005. Allometry of Alarm Calls: Black-Capped Chickadees Encode Information about Predator Size. Science 308 (5730): 1934-37.

Tigreros N, Davidowitz G. 2019. Flight-Fecundity Tradeoffs in Wing-Monomorphic Insects."Advances in Insect Physiology. https://doi.org/10.1016/bs.aiip.2019.02.001.

Tooker JF, Hauser M, Hanks LM. 2006. Floral Host Plants of Syrphidae and Tachinidae (Diptera) of Central Illinois. Annals of the Entomological Society of America 99 (1): 96-112. Welch $\mathrm{CH}$. 2006. Intraspecific competition for resources by Ormia depleta (Diptera: Tachinidae) larvae. Florida Entomologist 89(4): 497-502.

West GB, Brown JH, Enquist BJ. 1997. A General Model for the Origin of Allometric Scaling Laws in Biology. Science 276 (5309): 122-26.

West GB Brown JH, Enquis BJ. 1999. The Fourth Dimension of Life: Fractal Geometry and Allometric Scaling of Organisms. Science 284 (5420): 1677-79.

Wickman P, Karlsson B. 1989. "Abdomen Size, Body Size and the Reproductive Effort of Insects. Oikos 56 (2): 209.

Wilson, JK, Woods HA. 2015. Protection via Parasitism: Datura Odors Attract Parasitoid Flies, Which Inhibit Manduca Larvae from Feeding and Growing but May Not Help Plants.” Oecologia 179 (4): 1159-71.

Zera AJ, Brink T. 2000. Nutrient Absorption and Utilization by Wing and Flight Muscle Morphs of the Cricket Gryllus firmus: Implications for the Trade-off between Flight Capability and Early Reproduction. Journal of Insect Physiology 46 (8): 1207-18.

Zera AJ, Denno RF. 1997. Physiology and Ecology of Dispersal Polymorphism in Insects. 
497

498

499

500

501

502

503

504

505

506

507

508

509

510

511

512

513

514

515

516

517

518

519

520

521

522

523

524

525

526

527

528

529

Annual Review of Entomology 42: 207-30.

Zera AJ, Potts L, Kobus J. 1998. The Physiology of Life-History Trade-Offs: Experimental Analysis of a Hormonally Induced Life-History Trade-off in Gryllus assimilis. The American Naturalist 152 (1): 7-23.

Zera AJ, Harshman LG. 2001. The Physiology of Life History Trade-Offs in Animals. Annual Review of Ecology and Systematics 32 (1): 95-126.

\section{Figure Captions}

Figure 1: Adult Drino rhoeo weight as a function of competitive larval environment (cohort size) and host quality. (a) The negative relationship between increasing larval competition and adult size. (b) The positive relationship between host quality (head-capsule width) and adult fly weight. In both panels, green points represent male flies and red points female flies. Black lines represent the trendline for the best fit linear mixed effects model that included cohort size and head-capsule width as fixed effects and host as a random effect. Gray bands represent the $95 \%$ confidence interval. Raw data is jittered horizontally slightly to aid in visualization, and larger black points represent the predicted (based on the best fit models) adult fly weight for each host.

\section{Figure 2: Relative weight investment in different adult Drino rhoeo body structures as a} function of body size. Different panels show relationships of relative weight investment of (a) heads, (b) wings, (c) legs, (d) thoraces and (e) abdomens. Males flies are represented by green points whereas females are represented by red points. Solid lines depict the trendlines for the best fit model for each body structure whereas gray bands represent the $95 \%$ confidence interval. In panels with two lines depicted $(d-e)$, there was a significant difference between males and females.

\section{Figure 3: Energetic trade-offs in allocation to different body segments of adult Drino} rhoeo. (a) The percent calories of heads, abdomens and thoraces for male and female flies as a function of body size. Abdomens (red) and thoraces (blue) show significant differences between males (triangles) and females (circles), while there was no difference between males 
530 and females in energy allocation to heads (green). (b) Energy allocation trade-off between

531 abdomens and thoraces for Drino rhoeo. Individual abdomen and thorax energy content is

532 normalized for total energy content of all three main body segments and plotted against each

533 other. Female flies are in red and male flies are in green, while body size is represented by the

534 size of the circle. Marginal density plots depict the differences in the allocation of resources to

535 thoraces and abdomens between males and females.

536

537 Supplemental Figure 1: Allometric scaling relationships of the mass of thoraces, 538 abdomens, heads, legs and wings of adult Drino rhoeo flies. In both panels, the dotted line

539 depicts the 1:1 line. The top panel (a) depicts thoraces and abdomens, with trendlines and 95\%

540 confidence intervals plotted from OLS regression models that included the effects of sex,

541 additively. (b) Depiction of the scaling of heads, legs and wings with trendlines and $95 \%$

542 confidence intervals plotted from OLS regression models. In these tissues, there was no

543 significant difference between males and females.

544

545

546

547

548

549 


\section{Figure 1}

Adult Drino rhoeo weight as a function of competitive larval environment (cohort size) and host quality

(a)The negative relationship between increasing larval competition and adult size. (b) The positive relationship between host quality (head-capsule width) and adult fly weight. In both panels, green points represent male flies and red points female flies. Black lines represent the trendline for the best fit linear mixed effects model that included cohort size and headcapsule width as fixed effects and host as a random effect. Gray bands represent the 95\% confidence interval. Raw data is jittered horizontally slightly to aid in visualization, and larger black points represent the predicted adult fly weight for each host. 

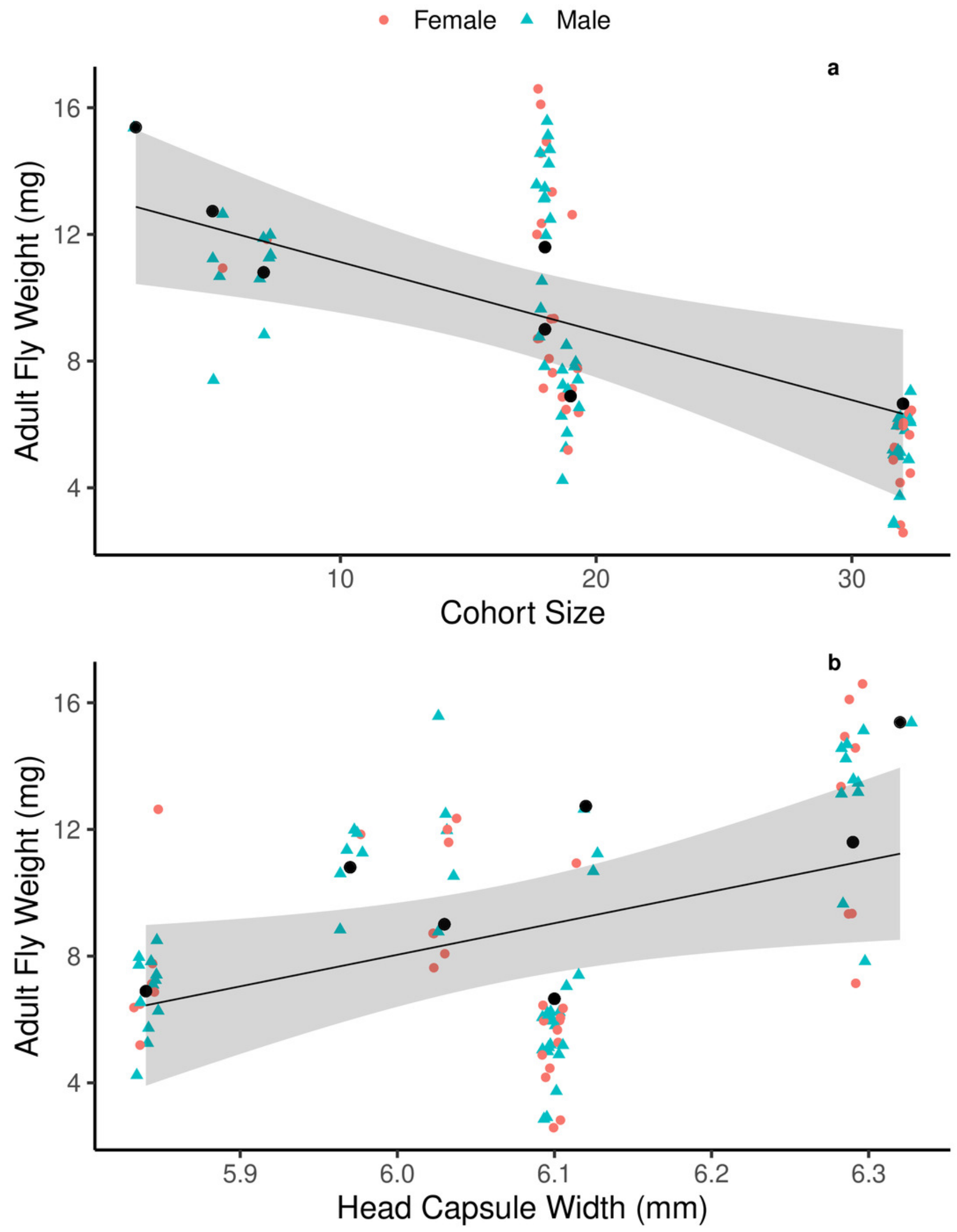


\section{Figure 2}

Relative weight investment in different adult Drino rhoeo body structures as a function of body size.

Different panels show relationships of relative weight investment of (a) heads,(b) wings, (c) legs, (d) thoraces and (e) abdomens. Males flies are represented by green dots whereas females are represented by red dots. Solid lines depict the trendlines for the best fit model for each body structure whereas gray bands represent the $95 \%$ confidence interval. In panels with two lines depicted $(d-e)$, there was a significant difference between males and females. 

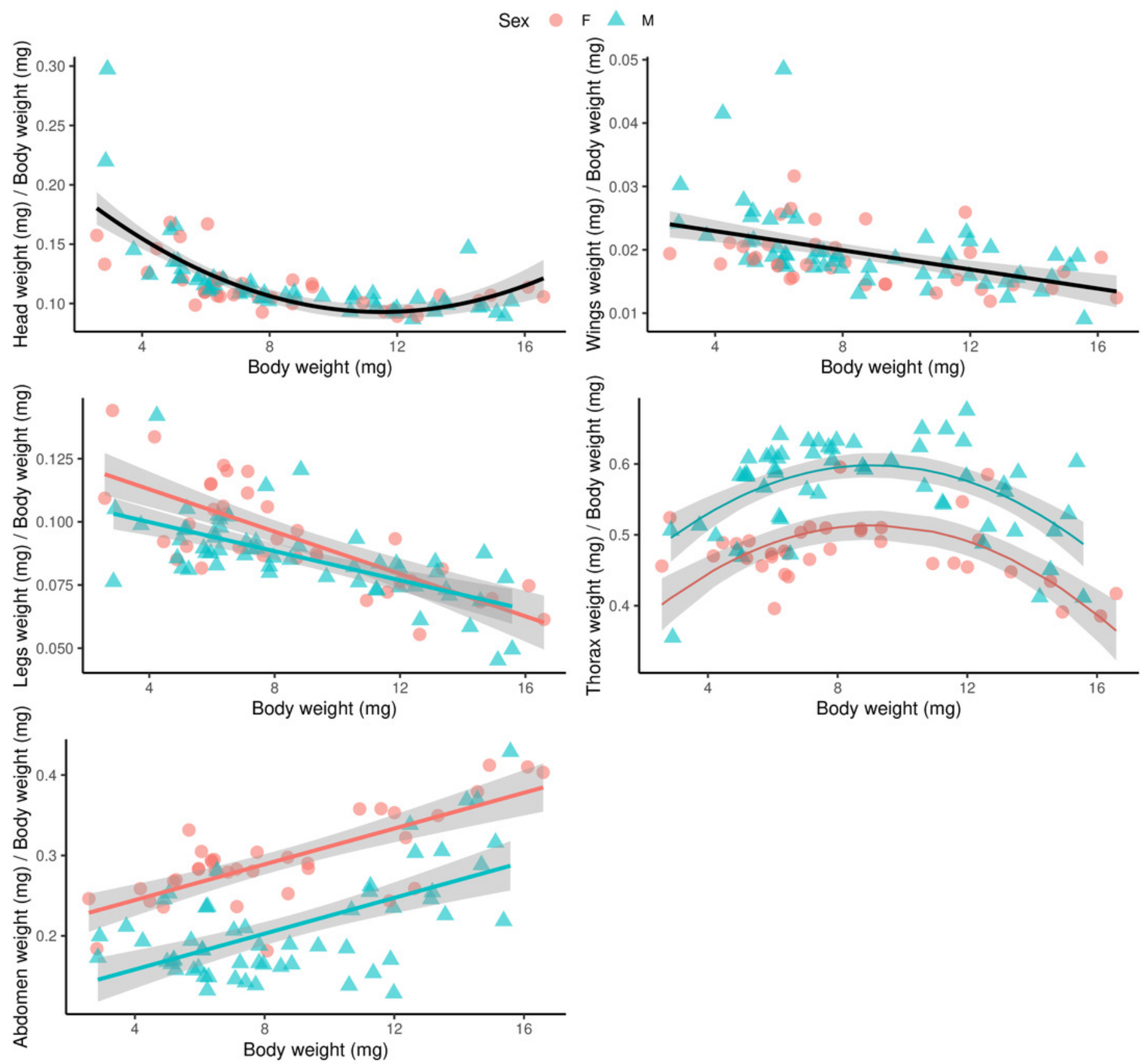


\section{Figure 3}

Energetic trade-offs in allocation to different body segments of adult Drino rhoeo.

(a) The percent calories of heads, abdomens and thoraces for male and female flies as a function of body size. Abdomens (red) and thoraces (blue) show significant differences between males (triangles) and females (circles), while there was no difference between males and females in energy allocation to heads (green). (b) Energy allocation trade-off between abdomens and thoraces for Drino rhoeo. Individual abdomen and thorax energy content is normalized for total energetic content of all three main body segments and plotted against each other. Female flies are in red and male flies are in green, while body size is represented by the size of the circle. Marginal density plots depict the differences in the allocation of resources to thoraces and abdomens between males and females. 

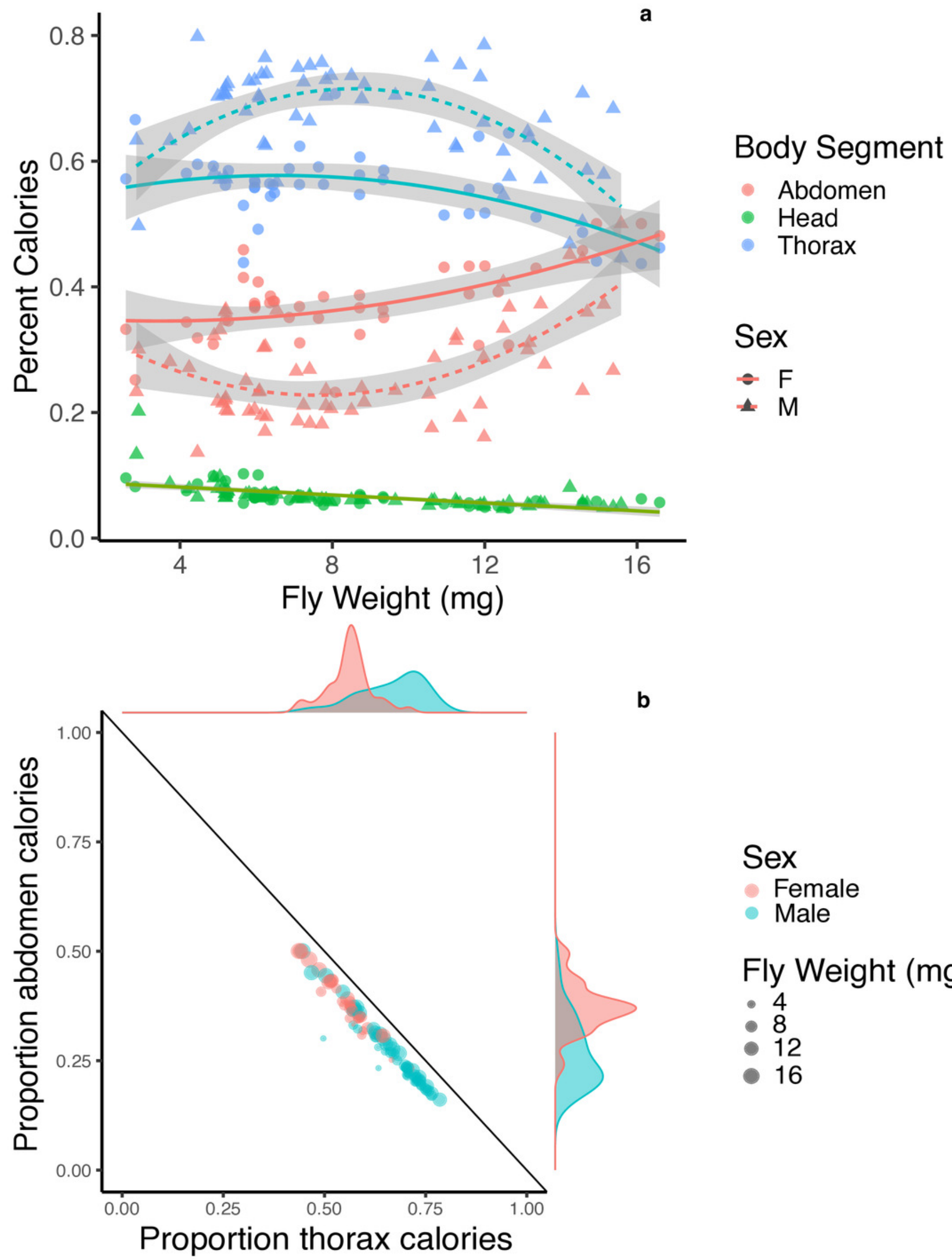

Sex

Female Male

Fly Weight (mg)

- 4

- 8

- 12

16 


\section{Table 1 (on next page)}

Model selection for factors affecting adult fly weight 
1

2

3

4

5

6

7

8

9

10

11

12

13

14

15

16

17

18

19

20

21

22

23

24

25

26

27

28

29

30

31

32

33

34

35

36

37

38

39

40

41 Table 1. Model Selection for Factors Affecting Adult Fly Weight

\begin{tabular}{l|l|l|l}
\hline Model & Marginal $R^{2}$ & Conditional $R^{2}$ & AIC \\
\hline Weight $\sim$ cohort size & 0.338 & 0.716 & 408
\end{tabular}

Peer) reviewing PDF | (2019:08:40077:3:0:NEW 17 Feb 2020) 


\begin{tabular}{l|l|l|l} 
Weight $\sim$ cohort size + head-capsule width & $\mathbf{0 . 4 6 2}$ & $\mathbf{0 . 6 8 3}$ & $\mathbf{4 0 1}$ \\
Weight $\sim$ cohort size * head-capsule width & 0.446 & 0.706 & 402 \\
Weight $\sim$ cohort size*head-capsule width ${ }^{*}$ Sex & 0.438 & 0.699 & 407 \\
\hline
\end{tabular}

42 


\section{Table 2 (on next page)}

Linear mixed effects model predicting fly weight as a function of cohort size and headcapsule width 
1

2

3 Table 2. Linear mixed effects model predicting fly weight as a function of cohort size and 4 head-capsule width

\begin{tabular}{|c|c|c|c|c|c|}
\hline \multicolumn{4}{|l|}{ Fixed Effects } & \multirow[b]{2}{*}{ t-value } & \multirow[b]{2}{*}{$p$-value } \\
\hline Parameter & Estimate & $\begin{array}{l}\text { Standard } \\
\text { Error }\end{array}$ & d.f. & & \\
\hline Intercept & -47.205 & 0.1909 & 85 & -1.723 & 0.09 \\
\hline Cohort size & -0.218 & 0.2727 & 4 & -3.000 & 0.04 \\
\hline $\begin{array}{l}\text { Head Capsule } \\
\text { Width }\end{array}$ & 9.972 & 0.2466 & 4 & 2.226 & 0.09 \\
\hline
\end{tabular}

Random Effects (Host - random intercepts)

\begin{tabular}{l|l|l}
\hline Parameter & Intercept & Residual \\
\hline StdDev & 0.1947 & 1.935 \\
\hline
\end{tabular}

5

6

7

8

9

10

11 